\title{
Burosumab nel trattamento della osteomalacia indotta da neoplasia
}

\author{
Salvatore Minisola ${ }^{1}$
}

Accettato: 8 gennaio 2021 / Pubblicato online: 17 marzo 2021

(c) The Author(s) 2021

\section{Commento a:}

Burosumab for the treatment of tumor-induced osteomalacia.

S.M. Jan de Beur, P.D. Miller, T.J. Weber, M. Peacock, K. Insogna, R. Kumar, F. Rauch, D. Luca, T. Cimms, M.S. Roberts, J. San Martin, T.O. Carpenter. J Bone Min Res (2020) doi:10.1002/jbmr.4233

L'osteomalacia indotta da neoplasia, chiamata anche osteomalacia oncogenica, è una rara malattia caratterizzata sul piano biochimico da ridotti livelli sierici di fosfato in rapporto a valori elevati o inappropriatamente normali di Fibroblast Growth Factor 23 (FGF23) [1]. Sul piano clinico si manifesta principalmente con fratture, pseudo-fratture e dolori muscoloscheletrici. In conseguenza della mancanza di sintomi tipici della malattia, la diagnosi definitiva è spesso posta dopo anni; nel frattempo il paziente è visitato da numerosi specialisti, senza successo.

Una volta individuata la sede, la resezione della neoplasia causa della malattia rappresenta la cura definitiva; infatti, alla guarigione consegue la normalizzazione dei parametri biochimici, la riparazione delle fratture, l'incremento dei valori densitometrici [2] e, infine, il miglioramento della qualità di vita dei pazienti.

Tuttavia, in alcuni casi, non è possibile individuare la neoplasia che secerne FGF-23, oppure essa è situata in sedi anatomiche difficili da resecare totalmente (quali ad esempio all'interno dei corpi vertebrali). Fino ad ora, in situa-

$凶$ S. Minisola

salvatore.minisola@uniroma1.it

1 Dipartimento di Scienze Cliniche, Internistiche, Anestesiologiche e Cardiovascolari, "Sapienza" Università di Roma, Roma, Italia zioni di questo genere, le uniche alternative farmacologiche erano costituite dalla somministrazione di fosfati e vitamina $\mathrm{D}$ attiva; tuttavia, è noto che la somministrazione di fosfati, oltre ad avere effetti indesiderati a livello gastrointestinale, stimolando la secrezione di paratormone può condurre alla comparsa di iperparatiroidismo terziario, descritto in questo tipo di malati. Alcuni tentativi effettuati con la somministrazione di octreotide, sulla base del rilievo di recettori per la somatostatina nella neoplasia hanno dato luogo a risultati contrastanti [3].

Il lavoro di Jan de Beur e collaboratori apre uno scenario importante nel trattamento dei pazienti con questa neoplasia nei casi in cui la localizzazione è risultata negativa oppure la completa resezione è impossibile. In uno studio di fase due, questi pazienti sono stati trattati con un anticorpo monoclonale diretto contro FGF23. Conseguentemente alla somministrazione del farmaco è stato possibile osservare un aumento dei livelli sierici di fosfato, unitamente al miglioramento degli indici istologici di osteomalacia; quest'ultimo rilievo è particolarmente importante poiché caratterizza a livello tissutale la guarigione della malattia. Il profilo di sicurezza è risultato accettabile e del tutto paragonabile a quello rilevato nel trattamento con questo farmaco dell'ipofosfatemia legata al cromosoma X (reazione locale nel sito di iniezione, ipersensibilità, aumento dei valori dei fosfati).

L'unico rilievo che potrebbe essere sollevato a questo lavoro è costituito dalla mancanza di un gruppo di controllo; tuttavia, considerando la rarità della malattia e i significativi aumenti ottenuti sul piano biochimico e istologico, questa obiezione può non essere discriminante.

In conclusione, questo studio fornisce le basi per il trattamento a lungo termine dell'osteomalacia oncogenica nei casi in cui il tumore non è stato individuato o la com- 
pleta escissione del tumore non è chirurgicamente fattibile. In assenza della terapia con burosumab, si dovrebbe ricorrere alla somministrazione dei farmaci tradizionali con gli effetti collaterali cui abbiamo precedentemente accennato.

Funding Note Open access funding provided by Università degli Studi di Roma La Sapienza within the CRUI-CARE Agreement.

Nota della casa editrice Springer Nature rimane neutrale in riguardo alle rivendicazioni giurisdizionali nelle mappe pubblicate e nelle affiliazioni istituzionali.

Open Access This article is licensed under a Creative Commons Attribution 4.0 International License, which permits use, sharing, adaptation, distribution and reproduction in any medium or format, as long as you give appropriate credit to the original author(s) and the source, provide a link to the Creative Commons licence, and indicate if changes were made. The images or other third party material in this article are included in the article's Creative Commons licence, unless indicated otherwise in a credit line to the material. If material is not included in the article's Creative Commons licence and your intended use is not permitted by statutory regulation or exceeds the permitted use, you will need to obtain permission directly from the copyright holder. To view a copy of this licence, visit http://creativecommons.org/licenses/by/4.0/.

\section{Bibliografia}

1. Minisola S, Peacock M, Fukumoto S et al (2017) Tumour-induced osteomalacia. Nat Rev Dis Primers 3:17044

2. Colangelo L, Pepe J, Nieddu L et al (2020) Long-term bone mineral density changes after surgical cure of patients with tumor-induced osteomalacia. Osteoporos Int 31(7):1383-1387

3. Paglia F, Dionisi S, Minisola S (2002) Octreotide for tumor-induced osteomalacia. N Engl J Med 346(22):1748-1749 